\title{
Validation of the Illness Perception Questionnaire for Schizophrenia in a German-Speaking Sample of Outpatients with Chronic Schizophrenia
}

\author{
Marialuisa Cavelti ${ }^{a}$ Giuliana Contin $^{a}$ Eva-Marina Beck ${ }^{a}$ Sara Kvrgic $^{a}$ \\ Joe Kossowsky ${ }^{b}$ Rolf-Dieter Stieglitz ${ }^{a}$ Roland Vauth ${ }^{a}$

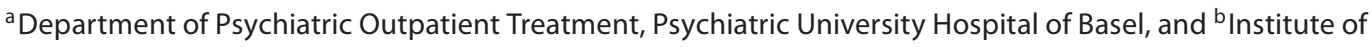 \\ Psychology, University of Basel, Basel, Switzerland
}

\section{Key Words}

Schizophrenia $\cdot$ Scale $\cdot$ Validity $\cdot$ Reliability $\cdot$ Illness

perception $\cdot$ Insight $\cdot$ Depression $\cdot$ Beliefs about

medication $\cdot$ Psychopathology

\begin{abstract}
Background: Because the mere definition of insight from the therapist's viewpoint may not be sufficient to identify treatment targets for adherence enhancement, we need assessment strategies which are more sensitive to the patient's perspective. Illness perception (IP), defined as the beliefs a patient holds about his/her health problems, has been shown to affect coping in the context of a physical or mental illness, e.g. compliance behaviour. To assess IP in people diagnosed with schizophrenia, the Illness Perception Questionnaire for Schizophrenia (IPQS) was developed. The aim of the present study was to analyse the psychometric properties of the German version of the IPQS. Sampling and Methods: The study sample consisted of 128 German-speaking outpatients suffering from chronic schizophrenia or schizoaffective disorder. To achieve comparability with the validation of the English scale version, the same constructs were assessed: psychopathology, depression, and beliefs about medication. Furthermore, insight into one's illness was assessed. Internal consistency, test-retest reliability and
\end{abstract}

\section{KARGER}

Fax +4161306 1234 E-Mail karger@karger.ch www.karger.com

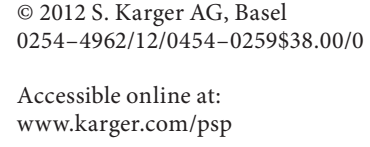

construct validity including convergent and discriminant validity were analysed. Results: Five of eight IPQS subscales were found to be internally reliable and all subscales demonstrated high stability over time. Correlations with validity measures indicated that the subscales assess dimensions of a construct, which is distinct from psychopathology, depression, beliefs about medication and insight, except for the Identity subscale which substantially overlapped with measures of insight. Conclusions: The German version of the IPQS is an essentially reliable and valid measure of IP for German-speaking people with a schizophrenia spectrum disorder. This may encourage its usage in further studies investigating the impact of subjective beliefs about mental health problems on outcome and recovery in schizophrenia. Copyright $\odot 2012$ S. Karger AG, Basel

\section{Introduction}

Although there are a number of effective interventions for people with a diagnosis of schizophrenia, such as antipsychotic medication, cognitive-behaviour therapy and family interventions [1], the outcomes with regard to

M.C. and G.C. contributed equally to the work. 
symptoms, social and vocational functioning, and wellness vary [2]. It is important to understand the causes for these variations in order to improve the situation for patients and their relatives.

A factor shown to explain significant amount of outcome variance in schizophrenia is non-compliance defined as the extent to which a person's behaviour corresponds to the recommendations of a healthcare provider [3]. Between 40 and $60 \%$ of patients with a schizophrenia spectrum disorder do not take their antipsychotic medication as prescribed $[4,5]$ and $24 \%$ of individuals with psychosis do not 'attend appointments as scheduled' [6]. In examining the reasons for non-compliance, lack of insight - defined as the unawareness of mental disorder, of symptoms, social consequences and need for treatment [7] - has been identified as a main risk factor $[4,8]$. Even if the significance of insight for compliance has been well demonstrated, and many reliable and valid assessments have been developed [9], the concept has been criticized due to its implicit paternalistic attitude: insight reflects the extent to which individuals agree to the biomedical illness model of schizophrenia [10].

In consequence, it was proposed to take the individual's subjective perspective in the context of illness into account more strongly, and the concept of illness perception (IP) was introduced. IP is defined as the self-definition of health status influenced by illness-related experiences in the past [11]. Originally developed in the physical health area, IP has recently been applied to individuals with mental disorders [12-15], their relatives [16], and mental health practitioners [17]. Crisp et al. [18] found that patients think differently about specific diagnoses, indicating the need for distinct assessment methods of IP. Additionally, Kinderman et al. [19] pointed out that while in the case of physical health problems, there may be a desire to identify a disease entity that can be diagnosed and separated from the sense of self, the distinction between the illness and the self in psychosis is often more blurred, making the creation of an illness model more difficult. In light of these arguments, Lobban et al. [20] adopted the Illness Perception Questionnaire (IPQ) and its newer versions [Illness Perception Questionnaire-Revised (IPQ-R), Brief Illness Perception Questionnaire (Brief IPQ); 21, 22], which were designed to assess IP in the context of physical health, to the special needs of patients with schizophrenia, resulting in the Illness Perception Questionnaire for Schizophrenia (IPQS). As with the IPQ and its newer versions, the IPQS is based on Leventhal et al.'s [23] self-regulation model which postulates two modes of illness representation: the first one is of a cognitive nature and encompasses beliefs about (1) the causes of the health problems, e.g. external agents including bacteria, viruses, job stress; internal sensitivity such as genetic factors; and behavioural aspects such as drinking alcohol or lack of sleep; (2) the identity of the health problems including symptoms and labels that define it; (3) the timeline (e.g. acute versus chronic); (4) the consequences of the health problems on one's life (e.g. social isolation, vocational difficulties or financial losses); and (5) the potential to control or cure the illness (e.g. by treatment or one's own behaviour). The second mode of illness representation is affective and includes direct emotional responses to health problems such as fear, anger or despair which are not mediated by cognitive appraisal [20]. Both representation modes together are thought to determine a person's reaction in the context of a health threat such as compliance behaviour as a kind of coping. In recognition of the essential role emotional dysregulation plays in mental illness, the consideration of both representation modes made the self-regulation model the most adequate model to adopt to schizophrenia in comparison to other common health belief models in the physical area [24]. The psychometric properties of the IPQS were demonstrated on a sample of English-speaking inpatients [20]. Primary evidence suggests that the IP assessed by the IPQS contributes to a better understanding of the individual differences in behavioural and emotional responses to schizophrenia which are not satisfactorily explained by differences in insight [25].

Whereas the IPQ for physical illnesses has already been translated into German [26; http://www.uib.no/ipq/ index.html], to the best of our knowledge, the IPQS has not. In order to use the IPQS in our research into the subjective factors influencing compliance and recovery in chronic schizophrenia, we translated the scale into German. The aim of the present study was to establish the psychometric properties of the German version, including internal consistency, test-retest reliability and construct validity, on a sample of outpatients. To achieve comparability with the validation of the English scale version [20], we assessed the same constructs as the original authors, and expected the IPQS subscales to correlate with measures of symptoms, depression and attitudes toward medication on a low level $(r<0.40)$, indicating that the IPQS assesses a distinct construct [discriminant validity; 27]. To the best of our knowledge, the associations of the IPQS subscales with measures of insight have not yet been examined. This is surprising given the fact that the Identity subscale assesses awareness and attribution of symptoms - two main components of the insight con- 
struct [7]. Therefore, we intended to investigate the associations between the IPQS subscales and measures of insight and hypothesized strong correlations $(\mathrm{r}>0.70)$ with Identity, indicating a high overlap of this dimension of IP with the insight construct [convergent validity; 27]. Because subjective and objective ratings of symptoms [28], depression [29], and insight [9] do not necessarily correlate with each other, we assessed each construct by both a patient-rated measure and a clinician-rated one, facilitating the separate analysis of their associations with the IPQS subscales.

\section{Methods}

\section{Recruitment and Procedure}

The recruitment took place at two community mental health services in Basel, Switzerland, between February 2009 and March 2010. All patients between 18 and 65 years of age and diagnosed with schizophrenia or schizoaffective disorder were asked for study participation. Exclusion criteria were a primary diagnosis of alcohol or substance dependency, an organic syndrome or learning disability, inadequate command of German and homelessness. Of the 236 individuals screened for participation, 144 $(61.02 \%)$ provided written informed consent after a full explanation of the study aims and procedures. The main reasons of refusal were suspiciousness ( $\mathrm{n}=33,35.9 \%)$, no interest $(\mathrm{n}=15$, $16.3 \%$ ) and fear of excessive demands or overwhelming emotions ( $\mathrm{n}=15,16.3 \%)$. All assessments including interviews and questionnaires were conducted by three research psychologists who underwent assessment training before the beginning of the study. Diagnoses were confirmed by the Structured Clinical Interview for Diagnostic and Statistical Manual of Mental Disorders - IV Axis I Disorders [30]. To avoid biases due to attention or motivation decline during the assessment, the presentation order of questionnaires was varied at random. Participants received a financial compensation of $40 \mathrm{CHF}$ in order to minimise selection bias by a high refuser rate. 128 participants $(88.89 \%)$ completed the assessment. Their data were used in the present study. 64 (50\%) completers agreed to fill out the questionnaires twice within a month for the purpose of test-retest reliability. They received an additional financial compensation of $50 \mathrm{CHF}$. The study was approved by the local ethics committee.

\section{Measurements}

The Illness Perception Questionnaire for Schizophrenia (IPQS)

The IPQS consists of 131 items which are rated on a 5-point Likert scale ( $1=$ 'strongly disagree' to $5=$ 'strongly agree') and is comprised of ten subscales:

- Identity (8 items): Originally, this subscale included 58 symptoms common in schizophrenia and schizoaffective disorder which were not further specified by the authors. The patients were asked to indicate which symptoms they experienced and to attribute them as due to 'mental health problems', 'side effects of my medication', and/or 'other factors'. Finally, the proportion of experiences attributed to each was calculated [20].
In order to reduce patient burden, we used - in contrast to the English version - eight symptoms of the Positive and Negative Syndrome Scale (PANSS) which were also assessed by the Scale to Assess Unawareness of Mental Disorder (SUMD): hallucinatory behaviour, delusions, conceptual disorganization, poor impulse control, lack of spontaneity and flow of conversation, blunted affect, poor attention, and few social contacts due to passive-apathetic withdrawal or anxious avoidance. This strategy guaranteed that participants were only asked to consider symptoms which were objectively present. If a symptom was judged to be present at the beginning of the study, as indicated by a PANSS score of 2 or above, patients were asked to indicate (1) whether they were aware of it ( 1 = 'strongly' to $5=$ 'not at all'), and if so (indicated by a score of 3 or lower), (2) whether they attributed it to mental health problems. We then calculated the relative proportion of experiences, which patients were aware of and attributed to mental health problems.

- Cause (26 items): A high item score denotes a high attribution of a cause to mental health problems, e.g. 'Chemical imbalance in the brain', 'My personality', 'Hereditary; it runs in my family', 'Money worries', 'Pollution in the environment', 'Alcohol', 'My upbringing', 'Thinking about things too much' or 'Someone spiked my drink with illicit drugs'. No subscale score was calculated.

- Timeline Acute/Chronic (6 items) and Timeline Cyclical (4 items): High scores denote a chronic and cyclical timeline of the mental health problems, respectively, e.g. 'I expect to have these mental health problems for the rest of my life' (Timeline Acute/Chronic) or 'Sometimes I have more symptoms than other times' (Timeline Cyclical).

- Consequences (11 items): A high subscale score denotes a perception of a high level of negative impact of the mental health problems on work, important relationships, family, social life, everyday functioning, and the perception of one's own interpersonal attractiveness, e.g. 'I have lost important relationships as a result of my mental health problems'. The subscale additionally includes an item assessing positive effects ('My mental health problems have had some positive effects on my life'; reversely scored).

- Personal Control (4 items): A high score denotes a high degree of perceived personal control over the mental health problems, e.g. 'There are some things that I can do to control my symptoms'.

- Personal Blame (3 items): A high score denotes a high degree of self-blame for the mental health problems, e.g. 'If I tried harder, I could control my symptoms'.

- Treatment Control (5 items): A high score denotes a belief that treatment will be helpful in managing mental health problems, e.g. 'My treatment can control my mental health problems'.

- Illness Coherence (5 items): A high score denotes a sense of not having a coherent understanding of the mental health problems, e.g. 'I don't have any understanding of my mental health problems at all'.

- Emotional Representation (9 items): A high score denotes a strong negative emotional response to the mental health problems, e.g. 'I get depressed when I think about my mental health'. 
All subscales - except for Personal Control and Personal Blame - demonstrated acceptable levels of internal consistency ( $\alpha$ $>0.70$ ) and high stability over time [20].

We used Lobban et al.'s [20] IPQS and translated it into German. For cross-cultural research, semantic and content equivalence are needed. To establish semantic equivalence of the German version of the IPQS, a back-translation method was used. After the original version was translated into German, a bilingual $\mathrm{PhD}$ student translated the German version back into English without referring to the original English instrument, in accordance with the recommended procedure for translating research instruments [31]. Next, we compared the original English version with the German back-translation version and developed the final version by consensus. For content equivalence, the bilingual $\mathrm{PhD}$ student evaluated the content of each item. All items were found to be relevant for IP. The German version of the instrument is available upon request from the corresponding author.

The Positive and Negative Syndrome Scale (PANSS)

The PANSS is a semi-structured interview to assess positive and negative symptoms as well as general psychopathology common in schizophrenia. In our study, their occurrence during the last seven days was evaluated. The 30 items are rated on a 7-point Likert scale ( $1=$ 'absent' to 7 = 'extreme') with higher scores indicating higher levels of psychopathology. By summing up single items, the three subscales Positive Syndrome (7 items), Negative Syndrome (7 items), and General Psychopathology (16 items) are formed [32]. The measure was shown to be reliable with Cronbach's $\alpha$ coefficients of 0.73 for the Positive Syndrome Scale, of 0.83 for the Negative Syndrome Scale, and of 0.79 for the General Psychopathology Scale. For the German version of the PANSS, an inter-rater reliability of $80 \%$ was demonstrated after a standardized rater training [33].

The Subjective Well-Being under Neuroleptic Treatment Scale - Short Form (SWN-K)

The SWN is a self-report instrument to evaluate subjective wellbeing under antipsychotic treatment with regard to the previous seven days. We used the short form consisting of 20 items and five subscales Mental Functioning (e.g. 'I find it easy to think'), SelfControl (e.g. 'I feel powerless and not in control of myself'), Emotional Regulation (e.g. 'I have no hope for the future'), Physical Functioning (e.g. 'My body feels familiar'), and Social Integration (e.g. 'I find it easy to keep in touch with people around me'). Patients are asked to rate their responses on a 6-point Likert scale (1 $=$ 'not at all' to $6=$ 'very much'). In our study we used only the total score with higher scores indicating a better subjective well-being during antipsychotic treatment. Based on data of a German sample, the short version was shown to correlate highly with the original scale indicating good construct validity and to be highly reliable with a Cronbach's $\alpha$ coefficient for the total score of 0.92 [34].

The Calgary Depression Scale for Schizophrenia (CDSS)

The CDSS is a semi-structured interview which was specifically developed to assess depression separately from negative symptoms in schizophrenia. It consists of eight questions and one interviewer observation which are all rated on a 4-point Likert scale $(0=$ 'absent' to $3=$ 'severe'). By summing up single items, a total score is built with a higher score indicating a higher level of depression. Construct validity was confirmed with other well- established measures of depression. Reliability of the German version has been demonstrated with an intra-class correlation coefficient (ICC) of 0.70 [35].

The Beck Depression Inventory-Revised (BDI-II)

The BDI-II is a self-report instrument to assess the intensity of depressive symptoms in persons with and without a clinical diagnosis of depression. It consists of 21 items, each with four statements indicating increasing severity (4-point Likert scale from 0 to 3). For example: 'In the past two weeks including today: I do not feel sad', 'I feel sad much of the time', 'I am sad all the time', 'I am so sad or unhappy that I cannot tolerate it'. By summing up single items, a total score is achieved ranging from 0 to 63 . Validity and reliability (internal consistency: $\geq 0.84$; test-retest reliability: $\geq 0.75$ ) of the German version of the BDI-II were established in clinical and non-clinical samples [36].

The Scale to Assess Unawareness of Mental Disorder (SUMD)

The SUMD is a semi-structured interview to evaluate global insight into illness and specific insight into symptoms with regard to the present and the past [37]. The instrument design allows for the independent usage of any subscale depending on the goal of the investigation. In our study, we used the three non-symptomatic summary items assessing (1) global awareness of mental disorder, (2) awareness of the achieved effects of medication, and (3) awareness of the social consequences of having a mental disorder with regard to the present (defined as the past 7 days). The items are rated on a 5 -point Likert scale ( $1=$ 'aware' to $5=$ 'unaware') with higher scores indicating poorer awareness. For the original English scale version, ICCs for the three summary items of 0.89 , 0.75 , and 0.68 were reported [37].

\section{The Insight Scale (IS)}

The IS is a self-report questionnaire to measure insight into psychiatric illness and treatment. The instrument contains eight statements to which the patient responds with 'I agree', 'I disagree', or 'I am unsure'. The items form the three subscales Relabelling Symptoms (e.g. 'Some of the symptoms were made by my mind'), Awareness of Illness (e.g. 'I am mentally well'), and Need for Treatment (e.g. 'I do not need medication'). Each subscore can reach a maximum of 4 with higher scores representing greater extent of insight. By summing up single items, a total score with a maximum of 12 indicating full insight is achieved. In our analyses, we used the total score. This was found to have a Cronbach's $\alpha$ coefficient of 0.75 and a test-retest reliability coefficient of 0.90 in an English-speaking sample [38].

The Beliefs about Medicines Questionnaire (BMQ)

The BMQ is an 18-item questionnaire measuring patients' attitudes towards medication. It comprises two sections: (1) The BMQ Specific is a 10-item scale which assesses patients' perceptions of their prescribed medication. Five items form the Specific Necessity subscale (e.g. 'My medicines protect me from becoming worse'), and five items the Specific Concerns subscale (e.g. 'I sometimes worry about the long-term effect of my medicines'). (2) The BMQ General is an 8-item scale, which assesses more general beliefs about medication. Four items build the General Harm subscale (e.g. 'Doctors place too much trust on medicines'), and four items the General Overuse subscale (e.g. 'Most medicines are ad- 
dictive'). Each item is rated on a 5-point Likert scale (1 = 'strongly disagree' to 5 = 'strongly agree'). By summing up single items, the four subscale scores are achieved with higher scores indicating a stronger endorsement of the construct being measured. For the four subscales of the original English version, internal consistencies ranging from $\alpha=63$ to 0.74 were reported $[39,40]$.

\section{Statistical Analyses}

In order to examine if the test-retest subsample differed from the other study participants regarding socio-demographic and clinical characteristics, we used t tests for continuous variables and $\chi^{2}$ tests for categorical variables.

With regard to reliability, internal consistency was calculated for all subscales with the exception of Identity and Cause because these subscales were not designed to assess a coherent dimension [20]. The subscales were classified as internally consistent if the Cronbach's $\alpha$ coefficients exceeded 0.70 [41]. Additionally, Spearman's correlations $\left(r_{s}\right)$ between the items within each subscale and Pearson's correlations ( $r$ ) between the subscales were calculated. The non-parametric correlation was used due to the non-normal distribution of single items. To ensure that the items of a subscale assess the same dimension of the construct but are not redundant, correlation coefficients between 0.20 and 0.40 are recommended $[42,43]$. Regarding the inter-subscale correlations, coefficients should not exceed 0.70 , denoting that the subscales assess different construct dimensions [27]. Test-retest reliability was analysed using Pearson's correlations for associations between the scores at each time point, and t tests for differences between them. It was not possible to analyse test-retest reliability of the Identity subscale because awareness and attribution of symptoms were estimated only once at the beginning of the study in the context of the PANSS interview. Significant correlation coefficients were interpreted in terms of effect sizes as follows: $\mathrm{r}<0.10$ as small, $\mathrm{r}=0.30$ as moderate, and $\mathrm{r}>0.50$ as high [44]

With regard to construct validity, Pearson's correlations between the IPQS subscales and the measures of symptom severity, depression, beliefs about medication and insight were evaluated. Correlation coefficients $\leq 0.40$ were considered as indicators of discriminant validity, i.e. the instruments compared assess different constructs [27]. Correlation coefficients $\geq 0.70$ were judged as indicators of convergent validity, i.e. the instruments compared assessed the same construct [27]. The validity measures with significant correlations were then used as independent variables for multiple regression analyses to determine if the relationships with the IPQS subscales persisted after simultaneous adjustment.

All data analyses were performed using the Statistical Package for the Social Sciences Windows, Version 17.0 (SPSS Inc., Chicago, Ill., USA). If not otherwise specified, an $\alpha$ level of 0.05 was applied to define significance.

\section{Results}

\section{Sample Characteristics}

Of the 128 participants, 41 (32\%) were female. The mean age was 44.31 years $(\mathrm{SD}=11.78)$. The majority of participants lived alone $(n=69 ; 53.9 \%)$ and had neither a stable partnership - defined as lasting three months or longer $(n=103 ; 80.5 \%)-$ nor children $(n=96 ; 75 \%)$. The mean years of education were $12.23(\mathrm{SD}=2.87)$. Considering that nine years of education are obligatory in Switzerland, the sample may be judged as poorly educated. With regard to the vocational situation, a minority of participants $(\mathrm{n}=8 ; 14.1 \%)$ had paid work; the remainder was in sheltered employment $(n=41 ; 32.1 \%)$ or without any employment $(\mathrm{n}=69 ; 53.9 \%)$. As a consequence, the vast majority of participants $(n=103 ; 80.5 \%)$ received a governmental disability annuity. 90 participants (70.3\%) were diagnosed with schizophrenia and 38 (29.7\%) with schizoaffective disorder. The mean illness duration was 17.83 years $(\mathrm{SD}=11.59)$. On average, participants had been hospitalized 8.45 times $(\mathrm{SD}=11.45)$ and had received 7.54 years $(\mathrm{SD}=6.40)$ of outpatient treatment. The majority $(n=120 ; 93.8 \%)$ were treated with antipsychotics: 113 patients $(88.3 \%)$ with at least one atypical medicine, one patient $(0.8 \%)$ with a typical medicine, and six patients $(4.75 \%)$ with a combination of an atypical medicine with a typical one.

The test-retest subsample $(n=64 ; 50 \%)$ did not differ from the other study participants regarding socio-demographic and clinical characteristics with the single exception of parenthood: participants of the test-retest subsample had significantly fewer children (15.63 vs. $34.38 \%$; $\mathrm{p}=0.014)$.

\section{IPQS: Descriptive Statistics}

Regarding the Cause subscale, 'stress or worry' were judged by a majority of participants $(\mathrm{n}=47 ; 36.7 \%)$ as an important reason for the onset of their mental health problems (mode $=5$; range of possible scorers: $1-5$, with higher scores indicating stronger agreement). 'Chance or bad luck', 'my family's behaviour', 'family problems', 'thinking about things too much', and 'my own behaviour' were mostly $(n=40-54 ; 31.3-42.2 \%)$ regarded as partly relevant (mode $=3$ ) for the development of mental health problems. All other reasons (e.g. 'a germ or virus', 'my personality', 'lack of friends or people who care about me', 'hereditary; it runs in my family', 'overwork', 'taking illicit drugs', 'chemical imbalance in the brain') were appraised by the majority ( $\mathrm{n}=33-100 ; 25.8-77.3 \%)$ as irrelevant for the onset of their mental health problems (mode $=1$ ).

Means and standard deviations of all other subscales are shown in table 1.

IPQS: Reliability

Internal Consistency

While Timeline Acute/Chronic, Consequences, Treatment Control and Emotional Representation met the crit- 
Table 1. Mean scores, mean inter-item correlations and Cronbach's $\alpha$ coefficients for the IPQS subscales

\begin{tabular}{|c|c|c|c|c|}
\hline IPQS subscale $(\mathrm{n}=128)$ & $\begin{array}{l}\text { Number } \\
\text { of items }\end{array}$ & Mean $\pm \mathrm{SD}^{\mathrm{a}}$ & $\begin{array}{l}\text { Mean inter-item } \\
\text { correlation }^{\mathrm{b}}\end{array}$ & $\alpha$ \\
\hline \multicolumn{5}{|l|}{ Identity $^{c}$} \\
\hline Symptom awareness $(\mathrm{n}=110)$ & 8 & $2.26 \pm 1.38$ & - & - \\
\hline Symptom attribution $(n=92)$ & 8 & $2.83 \pm 1.48$ & - & - \\
\hline Timeline acute/chronic & 6 & $3.30 \pm 0.08$ & 0.41 & 0.81 \\
\hline Timeline cyclical & 4 & $3.67 \pm 0.07$ & 0.35 & 0.67 \\
\hline Consequences & 11 & $3.10 \pm 0.06$ & 0.24 & 0.76 \\
\hline Personal control & 4 & $3.49 \pm 0.07$ & 0.24 & 0.52 \\
\hline Personal blame & 3 & $2.74 \pm 0.09$ & 0.26 & 0.51 \\
\hline Treatment control & 5 & $3.95 \pm 0.07$ & 0.40 & 0.74 \\
\hline Illness coherence ${ }^{\mathrm{d}}$ & 5 & $2.40 \pm 0.06$ & 0.18 & 0.48 \\
\hline Emotional representation & 9 & $2.79 \pm 0.08$ & 0.38 & 0.85 \\
\hline
\end{tabular}

ical value of $\alpha>0.70$ for internal consistency (see table 1 ), Timeline Cyclical $(\alpha=0.67)$, Personal Control $(\alpha=0.52)$, Personal Blame $(\alpha=0.51)$ and Illness Coherence $(\alpha=0.48)$ failed to reach it. Given that Timeline Cyclical contains only four items, which is likely to reduce internal consistency, the $\alpha$ score of 0.67 may be judged as acceptable. However, the subscales assessing personal control, personal blame and illness coherence failed to reach reliability.

\section{Inter-Item Correlations}

The correlations between the items within the subscales ranged between $r_{s}=0.24$ and $r_{s}=0.40$ (see table 1 ), indicating that they assess the same dimension of the construct but are not redundant. There were two exceptions, Illness Coherence $\left(r_{s}=0.18\right)$ and Timeline Acutel Chronic $\left(\mathrm{r}_{\mathrm{s}}=0.41\right)$, which both deviated just marginally from the recommended range for inter-item correlations between 0.20 and $0.40[42,43]$.

Inter-Subscale Correlations

We found significant correlations among the IPQS subscales which ranged from $r=0.22$ to $r=0.67$ (table 2). This indicates that the subscales assess dimensions of IP which are not redundant $[\mathrm{r}<0.70 ; 27]$.

Test-Retest Reliability

The subscales showed highly positive correlations over the test-retest period which ranged from $r=0.49$ to $r=$
0.73 (table 3). This indicates a strong association between the two assessment points [44]. Additionally, no significant differences were found between the scores assessed at the two time points.

\section{IPQS: Construct Validity}

The Pearson's correlation coefficients between the IPQS subscales and the validity measures are presented in table 4.

The significant correlation coefficients between the IPQS subscales and the validity measures ranged from $r=0.18$ to $r=0.63$, indicating a moderate to high relationship between the IP dimensions and symptom severity, depression, insight and beliefs about medication [44]. None of the found correlation coefficients exceeded the critical value for convergent validity $(r>0.70)$, denoting that the validity measures (PANSS, SWNK, CDSS, BDI-II, SUMD, IS, BMQ) do not assess the same construct as the IPQS. Moreover, most correlation coefficients lay below the critical value for discriminant validity $(r<0.40)$, indicating that the instruments compared assess different constructs [27]. However, there were some exceptions with correlation coefficients between 0.40 and 0.70 , suggesting a certain overlap between the constructs assessed: the subjective measure of psychopathology (SWN-K total score) with Consequences $(\mathrm{r}=-0.62)$, Illness Coherence $(\mathrm{r}=-0.46)$ and Emotional Representation ( $\mathrm{r}=-0.63$ ); the subjective measure of depression (BDI-II) with Consequences ( $\mathrm{r}=$ 
Table 2. Inter-subscale correlations ${ }^{\text {a }}$ for the IPQS

\begin{tabular}{|c|c|c|c|c|c|c|c|c|c|}
\hline & \multicolumn{2}{|l|}{ Identity $^{\mathrm{b}}$} & \multirow{2}{*}{$\begin{array}{l}\text { Timeline } \\
\text { acute/chronic } \\
(\mathrm{n}=128)\end{array}$} & \multirow{2}{*}{$\begin{array}{l}\text { Timeline } \\
\text { cyclical } \\
(\mathrm{n}=128)\end{array}$} & \multirow{2}{*}{$\begin{array}{l}\text { Conse- } \\
\text { quences } \\
(n=128)\end{array}$} & \multirow{2}{*}{$\begin{array}{l}\text { Personal } \\
\text { control } \\
(\mathrm{n}=128)\end{array}$} & \multirow{2}{*}{$\begin{array}{l}\text { Personal } \\
\text { blame } \\
(\mathrm{n}=128)\end{array}$} & \multirow{2}{*}{$\begin{array}{l}\text { Treatment } \\
\text { control } \\
(\mathrm{n}=128)\end{array}$} & \multirow{2}{*}{$\begin{array}{l}\text { Illness } \\
\text { coherence }^{c} \\
(\mathrm{n}=128)\end{array}$} \\
\hline & $\begin{array}{l}\text { awareness } \\
(\mathrm{n}=110)\end{array}$ & $\begin{array}{l}\text { attribution } \\
(\mathrm{n}=92)\end{array}$ & & & & & & & \\
\hline Timeline acute/chronic & $-0.30^{* *}$ & $-0.37^{* *}$ & & & & & & & \\
\hline Timeline cyclical & -0.18 & $-0.26^{*}$ & $0.43^{* *}$ & & & & & & \\
\hline Consequences & $-0.33^{* *}$ & $-0.27^{* *}$ & $0.38^{* *}$ & $0.47^{* *}$ & & & & & \\
\hline Personal control & 0.01 & $-0.24^{*}$ & 0.01 & 0.16 & 0.09 & & & & \\
\hline Personal blame & 0.01 & $0.25^{*}$ & -0.12 & 0.14 & 0.07 & 0.08 & & & \\
\hline Treatment control & -0.08 & -0.20 & 0.12 & $0.23^{* *}$ & -0.16 & $0.32^{* *}$ & 0.01 & & \\
\hline Illness coherence ${ }^{c}$ & -0.03 & -0.00 & $0.21^{*}$ & 0.12 & $0.23^{* *}$ & $-0.29^{* *}$ & $0.22^{*}$ & $-0.36^{* *}$ & \\
\hline Emotional representation & $-0.27^{* *}$ & -0.17 & $0.40^{* *}$ & $0.42^{* *}$ & $0.67^{* *}$ & -0.04 & $0.21^{*}$ & -0.16 & $0.46^{* *}$ \\
\hline $\begin{array}{l}\text { a Pearson's correlation } \\
\text { b Higher scores indicat } \\
{ }^{c} \text { Higher scores denote } \\
{ }^{*} \mathrm{p}<0.05 \text {; }{ }^{* *} \mathrm{p}<0.01 .\end{array}$ & $\begin{array}{l}\text { er awarenes } \\
\text { oherent und }\end{array}$ & $\begin{array}{l}\text { s of symptom } \\
\text { derstanding o }\end{array}$ & $\begin{array}{l}\text { espectively, lowe } \\
\text { e mental health }\end{array}$ & $\begin{array}{l}\text { attributio } \\
\text { roblems. }\end{array}$ & to mental & lth proble & & & \\
\hline
\end{tabular}

0.45 ) and Emotional Representation ( $\mathrm{r}=0.47$ ); the measures of insight (IS, SUMD) with Identity $(\mathrm{r}=-0.41$ to 0.61$)$ and Timeline Acute/Chronic $(\mathrm{r}=0.47)$; and the measures of beliefs about medication with Consequences $(\mathrm{r}=0.53)$, Treatment Control $(\mathrm{r}=-0.44$ to -0.52) and Emotional Representation $(\mathrm{r}=0.47)$.

The following significant associations among the IPQS subscales and validity measures persisted after controlling for inflated $\alpha$ errors due to multiple correlations by conducting regression analyses (table 5 ): a stronger attribution of present symptoms to mental health problems (Identity) was associated with more awareness of the mental disorder $(\beta=0.37, p=0.003)$ and its social consequences ( $\beta=0.25, p=0.016$ ) according to the clinicians' perspective (SUMD). The perception of a more chronic and cyclical timeline was related to higher levels of insight assessed by patients' ratings (IS; $\beta=0.35, \mathrm{p}=$ 0.001 , respectively $\beta=0.21, p=0.040$ ) and, additionally, to more positive beliefs about the prescribed medication with regard to its necessity $(\beta=0.22, p=0.018$, respectively $\beta=0.22, p=0.025$ ). More perceived negative consequences of the mental health problems were associated with less subjective well-being $(\beta=-0.39, \mathrm{p}<0.001)$ and more concerns about the prescribed medication $(\beta=$ $0.27, \mathrm{p}=0.005$ ). Higher levels of self-blame were linked to lower levels of insight into the negative consequences of the illness according to clinicians' ratings (SUMD; $\beta=0.18, p=0.046$ ). A stronger belief in treatment control was associated with lower levels of general psychopathol$\operatorname{ogy}(\beta=-0.20, p=0.041)$, higher levels of subjective wellbeing ( $\beta=0.36, p=0.001)$, higher levels of insight ac-
Table 3. Test-retest reliability based on a 4 -week test interval

\begin{tabular}{lll}
\hline IPQS subscale & Correlations $(\mathrm{r})^{\mathrm{a}}$ & Differences $(\mathrm{T})^{\mathrm{b}}$ \\
\hline Timeline acute/chronic & $0.70^{*}$ & 0.38 \\
Timeline cyclical & $0.63^{*}$ & 0.72 \\
Consequences & $0.71^{*}$ & 0.54 \\
Personal control & $0.49^{*}$ & 0.53 \\
Personal blame & $0.63^{*}$ & 0.72 \\
Treatment control & $0.63^{*}$ & 0.34 \\
Illness coherence & $0.65^{*}$ & 0.14 \\
Emotional representation & $0.73^{*}$ & -0.81 \\
\hline
\end{tabular}

a Pearson's correlation.

$\mathrm{b}$ t test score.

c Higher scores denote less coherent understanding of the mental health problems.

${ }^{*} \mathrm{p}<0.05$.

cording to patients' ratings (IS; $\beta=0.22, \mathrm{p}=0.029$ ) and more positive beliefs about the prescribed medication $(\beta=0.32, p<0.001)$. A stronger sense of illness coherence was linked to higher levels of subjective well-being $(\beta=-0.36, p=0.002)$. Finally, a stronger emotional response to the mental health problems was related to lower levels of subjective well-being $(\beta=-0.40, p<0.001)$, higher levels of insight according to patients' ratings (IS; $\beta=0.22, p=0.017)$ and more concerns about the prescribed medication $(\beta=0.20, \mathrm{p}=0.041)$. The validity measures explained between 3 and $49 \%$ of the variance of the IPQS subscales. 
Table 4. Correlations ${ }^{\text {a }}$ between the IPQS subscales and subscales of the PANSS, SWN-K, CDSS, BDI, IS, SUMD and BMQ

\begin{tabular}{|c|c|c|c|c|c|c|c|c|c|c|}
\hline & \multicolumn{2}{|l|}{ Identity $^{\mathrm{b}}$} & \multirow{2}{*}{$\begin{array}{l}\text { Timeline } \\
\text { acute/ } \\
\text { chronic } \\
(\mathrm{n}=128)\end{array}$} & \multirow{2}{*}{$\begin{array}{l}\text { Timeline } \\
\text { cyclical } \\
(\mathrm{n}=128)\end{array}$} & \multirow{2}{*}{$\begin{array}{l}\text { Conse- } \\
\text { quences } \\
(n=128)\end{array}$} & \multirow{2}{*}{$\begin{array}{l}\text { Personal } \\
\text { control } \\
(\mathrm{n}=128)\end{array}$} & \multirow{2}{*}{$\begin{array}{l}\text { Personal } \\
\text { blame } \\
(\mathrm{n}=128)\end{array}$} & \multirow{2}{*}{$\begin{array}{l}\text { Treat- } \\
\text { ment } \\
\text { control } \\
(\mathrm{n}=128)\end{array}$} & \multirow{2}{*}{$\begin{array}{l}\text { Illness } \\
\text { coher- } \\
\text { ence }^{\mathrm{c}} \\
(\mathrm{n}=128)\end{array}$} & \multirow{2}{*}{$\begin{array}{l}\text { Emotional } \\
\text { represen- } \\
\text { tation } \\
(\mathrm{n}=128)\end{array}$} \\
\hline & $\begin{array}{l}\text { awareness } \\
(\mathrm{n}=110)\end{array}$ & $\begin{array}{l}\text { attribution } \\
(\mathrm{n}=92)\end{array}$ & & & & & & & & \\
\hline PANSS positive syndrome & 0.06 & $0.26^{*}$ & 0.03 & 0.02 & 0.13 & $-0.30^{* *}$ & 0.17 & $-0.24^{* *}$ & 0.17 & $0.23^{*}$ \\
\hline PANSS negative syndrome & 0.06 & $0.25^{*}$ & 0.02 & -0.02 & -0.07 & $-0.25^{* *}$ & 0.12 & -0.15 & 0.04 & -0.02 \\
\hline PANSS general psychopathology & -0.03 & $0.23^{*}$ & 0.11 & 0.08 & 0.17 & $-0.31^{* *}$ & 0.15 & $-0.38^{* *}$ & $0.23^{* *}$ & $0.29^{* *}$ \\
\hline SWN-K total score & $0.26^{* *}$ & $0.22 *$ & $-0.33^{* *}$ & $-0.19^{*}$ & $-0.62^{* *}$ & 0.15 & -0.03 & $0.34^{* *}$ & $-0.46^{* *}$ & $-0.63^{* *}$ \\
\hline CDSS & -0.17 & -0.19 & $0.19^{*}$ & $0.21^{*}$ & $0.26^{* *}$ & $-0.19^{*}$ & -0.05 & -0.08 & 0.05 & $0.25^{* *}$ \\
\hline BDI-II & $-0.20^{*}$ & -0.20 & $0.26^{* *}$ & $0.28^{* *}$ & $0.45^{* *}$ & $-0.20^{*}$ & 0.02 & $-0.20^{*}$ & $0.36^{* *}$ & $0.47^{* *}$ \\
\hline IS total score & $-0.41^{* *}$ & $-0.46^{* *}$ & $0.47^{* *}$ & $0.32^{* *}$ & $0.24^{* *}$ & 0.11 & -0.04 & $0.35^{* *}$ & 0.11 & $0.29^{* *}$ \\
\hline SUMD illness ${ }^{\mathrm{d}}$ & $0.45^{* *}$ & $0.61^{* *}$ & $-0.28^{* *}$ & -0.12 & -0.15 & -0.16 & 0.12 & $-0.20^{*}$ & 0.03 & -0.06 \\
\hline SUMD medication $^{\mathrm{d}}$ & $0.37^{* *}$ & $0.51^{* *}$ & $-0.21^{*}$ & -0.11 & -0.15 & -0.12 & 0.08 & $-0.30^{* *}$ & -0.05 & -0.15 \\
\hline SUMD consequences $^{\mathrm{d}}$ & $0.47^{* *}$ & $0.58^{* *}$ & $-0.23^{* *}$ & -0.12 & $-0.24^{* *}$ & -0.15 & $0.18^{*}$ & $-0.18^{*}$ & 0.04 & $-0.20^{*}$ \\
\hline BMQ specific necessity & $-0.26^{* *}$ & $-0.33^{* *}$ & $0.38^{* *}$ & $0.28^{* *}$ & 0.03 & 0.01 & -0.10 & $0.47^{* *}$ & 0.01 & 0.03 \\
\hline BMQ specific concerns & -0.10 & 0.04 & 0.01 & $0.18^{*}$ & $0.53^{* *}$ & -0.05 & 0.09 & $-0.34^{* *}$ & $0.31^{* *}$ & $0.47^{* *}$ \\
\hline BMQ general overuse & 0.00 & 0.19 & -0.04 & 0.05 & $0.31^{* *}$ & -0.02 & 0.10 & $-0.44^{* *}$ & $0.18^{*}$ & $0.23^{* *}$ \\
\hline BMQ general harm & 0.01 & $0.28^{* *}$ & -0.16 & -0.07 & $0.25^{* *}$ & -0.14 & 0.12 & $-0.52^{* *}$ & 0.16 & $0.24^{* *}$ \\
\hline
\end{tabular}

PANSS = Positive and Negative Syndrome Scale; SWN-K = Subjective Well-being under Neuroleptic treatment scale - short form; CDSS = Calgary Depression Rating Scale for Schizophrenia; BDI-II = Beck's Depression Inventory-Revised; IS = Insight Scale; SUMD = Scale to Assess Unawareness of Mental Disorder; BMQ = Beliefs about Medicines Questionnaire. a Pearson's correlation (r). ${ }^{\mathrm{b}}$ Higher scores indicate lower awareness of symptoms, respectively, lower attribution to mental health problems. ${ }^{c}$ Higher scores denote less coherent understanding of the mental health problems. ${ }^{\mathrm{d}}$ Higher scores indicate lower insight.

Table 5. Predictive validity of the IPQS subscales ${ }^{\mathrm{a}}$

\begin{tabular}{|c|c|c|c|c|c|c|c|c|c|c|}
\hline \multirow[t]{2}{*}{ Predictor variables } & \multicolumn{2}{|l|}{ Identity $^{\mathrm{b}}$} & \multirow{2}{*}{$\begin{array}{l}\text { Timeline } \\
\text { acute/ } \\
\text { chronic } \\
(\mathrm{n}=128)\end{array}$} & \multirow{2}{*}{$\begin{array}{l}\text { Timeline } \\
\text { cyclical } \\
(\mathrm{n}=128)\end{array}$} & \multirow{2}{*}{$\begin{array}{l}\text { Conse- } \\
\text { quences } \\
(\mathrm{n}=128)\end{array}$} & \multirow{2}{*}{$\begin{array}{l}\text { Personal } \\
\text { control } \\
(\mathrm{n}=128)\end{array}$} & \multirow{2}{*}{$\begin{array}{l}\text { Personal } \\
\text { blame } \\
(\mathrm{n}=128)\end{array}$} & \multirow{2}{*}{$\begin{array}{l}\text { Treat- } \\
\text { ment } \\
\text { control } \\
(\mathrm{n}=128)\end{array}$} & \multirow{2}{*}{$\begin{array}{l}\text { Illness } \\
\text { cohe- } \\
\text { rence } \\
(n=128)\end{array}$} & \multirow{2}{*}{$\begin{array}{l}\text { Emotional } \\
\text { represen- } \\
\text { tation } \\
(\mathrm{n}=128)\end{array}$} \\
\hline & $\begin{array}{l}\text { awareness } \\
(\mathrm{n}=110)\end{array}$ & $\begin{array}{l}\text { attribution } \\
(\mathrm{n}=92)\end{array}$ & & & & & & & & \\
\hline PANSS positive syndrome & & 0.08 & & & & -0.19 & & 0.09 & & 0.12 \\
\hline PANSS negative syndrome & & 0.15 & & & & -0.14 & & & & \\
\hline PANSS general psychopathology & & -0.03 & & & & -0.09 & & $-0.20^{*}$ & 0.08 & 0.02 \\
\hline SWN-K total score & 0.14 & 0.14 & -0.19 & 0.14 & $-0.39 * * *$ & & & $0.36^{* *}$ & $-0.36^{* *}$ & $-0.40^{* * *}$ \\
\hline CDSS & & & 0.13 & 0.17 & 0.06 & -0.07 & & & & 0.04 \\
\hline BDI-II & 0.00 & & 0.03 & 0.18 & -0.00 & -0.07 & & 0.02 & 0.06 & -0.01 \\
\hline IS total score & -0.14 & -0.01 & $0.35^{* *}$ & $0.21^{*}$ & 0.10 & & & $0.22 *$ & & $0.22 *$ \\
\hline SUMD illness ${ }^{\mathrm{d}}$ & 0.21 & $0.37^{* *}$ & -0.11 & & & & & 0.20 & & \\
\hline SUMD medication $^{\mathrm{d}}$ & -0.05 & -0.04 & 0.12 & & & & & -0.06 & & \\
\hline SUMD consequences $^{\mathrm{d}}$ & 0.24 & $0.25^{*}$ & 0.06 & & -0.10 & & $0.18^{*}$ & -0.08 & & -0.03 \\
\hline BMQ specific necessity & -0.04 & 0.01 & $0.22^{*}$ & $0.22^{*}$ & & & & $0.32^{* * *}$ & & \\
\hline BMQ specific concerns & & & & 0.17 & $0.27^{* *}$ & & & 0.10 & 0.09 & $0.20^{*}$ \\
\hline BMQ general overuse & & & & & 0.12 & & & -0.14 & & -0.01 \\
\hline BMQ general harm & & 0.15 & & & -0.06 & & & -0.14 & -0.04 & 0.05 \\
\hline $\mathrm{R}^{2}$ & 0.29 & 0.48 & 0.33 & 0.22 & 0.47 & 0.15 & 0.03 & 0.49 & 0.23 & 0.48 \\
\hline
\end{tabular}

PANSS = Positive and Negative Syndrome Scale; SWN-K = Subjective Well-being under Neuroleptic treatment scale - short form; CDSS = Calgary Depression Rating Scale for Schizophrenia; BDI-II = Beck's Depression Inventory-Revised; IS = Insight Scale; SUMD = Scale to Assess Unawareness of Mental Disorder; BMQ $=$ Beliefs about Medicines Questionnaire. a Values between dependent and independent variables are standardized $\beta$ coefficients. ${ }^{b}$ Higher scores indicate lower awareness of symptoms, respectively, lower attribution to mental health problems. ${ }^{c}$ Higher scores denote less coherent understanding of the mental health problems. ${ }^{\mathrm{d}} \mathrm{High}-$ er scores indicate lower insight.

${ }^{*} \mathrm{p}<0.05 ;{ }^{* *} \mathrm{p}<0.01 ;{ }^{* *} \mathrm{p}<0.001$. 


\section{Discussion}

\section{Summary and Implications}

In summary, the present results indicate that the analysed German version of the IPQS has acceptable psychometric properties regarding internal consistency, test-retest reliability and construct validity in the majority of subscales. While its adequacy for usage with inpatients suffering from schizophrenia or schizoaffective disorder has already been demonstrated [20], the present results suggest that the IPQS also adequately assesses outpatients' IP.

The individuals examined generally perceived their mental health problems as being chronic and cyclical, with many negative consequences for their life. Most people believed that they have some control over their illness and that treatment could help. They blamed themselves to a fair degree for their difficulties and experienced some negative emotions with regard to their mental health problems. They estimated the understanding of their mental health problems as rather incoherent. The subscales' mean scores were comparable with those reported for the English scale version [20]. With regard to beliefs about the causes of the mental health problems, we found 'stress and worry' to be the most accepted causal item. This result is also in line with Lobban et al.'s [20] work and is consistent with the stress vulnerability model of schizophrenia; the most widely accepted framework of psychosis [45]. However, it is highly relevant that items assessing factors which may contribute to the biological vulnerability proposed by the model (e.g. 'hereditary' or 'chemical imbalance in the brain') were not judged as relevant for illness causation in the present sample. This may indicate that our predominant strategy in psycho-education programs to highlight the biological vulnerability does not reach the majority of patients.

With regard to reliability, the majority of subscales demonstrated acceptable levels of internal consistency. It is possible that Personal Blame, Personal Control, and Illness Coherence did not reach the critical value of $\alpha=0.70$ due to the small number of items and the low correlations among them $\left(\mathrm{r}_{\mathrm{s}}=0.18-0.26\right)$. The reliability scores of Personal Blame and Personal Control had also been insufficient in the original English scale version [20]. The significant but moderate correlations $(\mathrm{r}<0.70)$ between the subscales $(r=0.22-0.67)$, which are comparable to the range of inter-subscale correlations reported by the original authors [20], indicate that they measure distinct dimensions of IP and are largely independent of each other.
Similar to the original scale [20], test-retest reliability for the German version was high for all subscales, indicating good stability over time.

In sum, with regard to validity, the pattern of correlations found between the IPQS subscales and the measures of symptoms, depression, beliefs about medication and insight denotes adequate construct validity of the German version of the IPQS.

The present study is the first to examine the relationships between measures of insight and the IPQS subscales. The high correlations between the insight measures (IS, SUMD) and the Identity subscale $(\mathrm{r}=0.37-0.61)$ may indicate that Identity assesses a component of IP which strongly overlaps with the insight construct. However, in contrast to our hypothesis the correlation coefficients failed to reach the critical value for convergent validity $(>0.70)$. The correlation coefficients between the insight measures and the other IPQS subscales were considerably lower $(\mathrm{r}=0.18-0.47)$, indicating that IP exceeds the insight construct in several ways (e.g. anticipated course of illness or expected controllability) and should therefore also be assessed in patients with low insight. In our opinion, the results indicate that the IP construct assesses the patients' point of view in a more comprehensive way than the insight construct, thus enabling clinicians to adopt treatment approaches of patients with schizophrenia to their individual needs. However, one result regarding the relationships between insight and dimensions of IP appeared to be contradictory, at least at first glance: while overall, measures of insight were not associated with the Personal Blame subscale, being more aware of the social consequences of having a mental disorder (SUMD subscale) correlated significantly with lower levels of personal blame. It is possible that by becoming aware of negative social consequences of the mental disorder, one begins to recognize that illness and recovery do not occur in a social vacuum but through the dynamic interplay between the individual and his/her environment [46]. Such a perception is self-protective and may result in lower self-blame. However, given the inadequate internal consistency of the Personal Blame subscale $(\alpha=$ 0.51 ) and the fact that only one single insight variable was significantly associated with the subscale, the result should probably be regarded as a methodological artefact.

We found a significant positive association between the Specific Necessity subscale of the BMQ and the Treatment Control subscale of the IPQS. Lobban et al. [20] also reported a positive correlation between Treatment Control and the measure of attitudes towards medica- 
tion (Drug Attitude Inventory, DAI). While positive beliefs about the prescribed medication have been shown to promote compliance with antipsychotic medication, negative beliefs seem to weaken it [40]. Thus, the present result suggests that medication compliance may be greater in people who believe that the pharmacological treatment will help them to control their illness. Additionally, the significant positive correlation between the Specific Concerns subscale of the BMQ and the Negative Consequences subscale of the IPQS may indicate that negative effects of antipsychotic medication on different aspects of interpersonal attractiveness, e.g. feeling reduced to the role of the patient by daily medication intake or experiencing stigmatizing side effects like immense weight gain, may contribute to medication nonadherence. Even if these relationships are currently of speculative nature and require further investigation, they support the idea of IP as a worthwhile predictor of compliance behaviour in patients with schizophrenia.

In contrast to the original study [20], the inclusion of both patient-rated and clinician-rated validity measures in our study enabled us to analyse differences in their associations with the IPQS subscales. For example, according to the patients' rating (BDI), but not according to the clinicians' rating (CDSS), feeling more depressed was linked to a stronger awareness of symptoms (Identity) - a main element of the insight-construct - as well as to less belief in treatment control. Because the causal relationship remains unclear due to the correlational analyses and cross-sectional nature of data, this result may be interpreted as preliminary evidence for both the idea that insight into one's mental illness and its consequences can have a demoralizing effect, as well as the hypothesis that depression causes people to evaluate their world more realistically, resulting in better insight [9]. In our research group, we found evidence for the first assumption by demonstrating that insight is associated with demoralization, mediated by less belief in treatment control [47]. Taken together, the different associations of patient- and clinician-rated measures with the IPQS subscales indicate the benefit of considering both subjective and objective variables in order to understand a patient's individual illness model.

\section{Limitations and Conclusion}

The present results are restricted by the following limitations: in our study, we decided to ask for awareness of symptoms and their attribution to mental health problems (Identity) only if a symptom was judged to be actually present by a clinician. This time-saving strategy was chosen to reduce patient burden which may arise if a full list of symptoms common to occur in schizophrenia is presented (58 symptoms in the original English scale version). Additionally, misattribution may only be meaningfully judged if there are symptoms present which the patient could misattribute. Otherwise, this strategy prevented the uncovering of differences in clinicians' and patients' views of exceptional experiences as belonging to mental health problems, because patients were allowed to evaluate only those symptoms which had been confirmed by clinicians. A further disadvantage of the applied strategy is that the results based on the German IPQS version are comparable to those based on the original English version - in all subscales except for Identity. Additionally, because we assessed the Identity subscale only once, we were not able to establish its test-retest reliability. Furthermore, the proposed structure of the IPQS has not been examined so far. For this purpose, further studies are needed which should preferably use confirmatory factor analysis, as it has been done for the IPQ for physical illnesses [17, 48, 49].

Nevertheless, the present results may encourage the use of the German version of the IPQS (especially both Timeline, Consequences, Treatment Control, and Emotional Representation subscales) in further studies investigating the impact of subjective beliefs about mental health problems on outcome variables such as illness and treatment course, or compliance behaviour in patients diagnosed with a schizophrenia spectrum disorder.

References

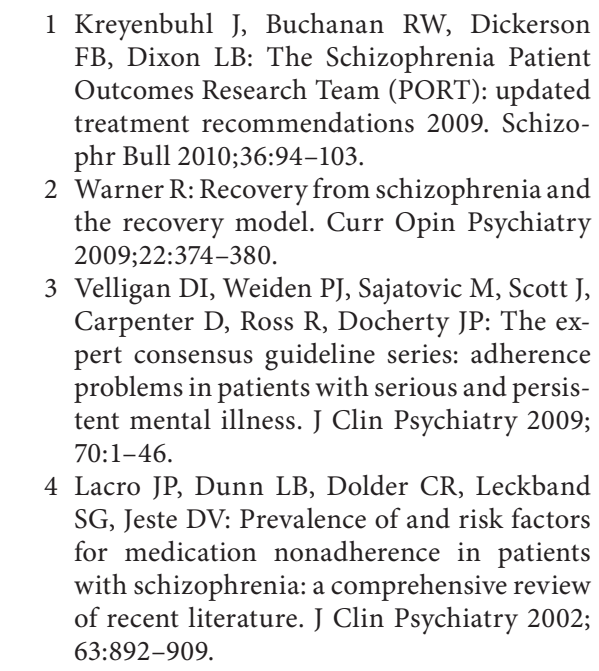

Cavelti/Contin/Beck/Kvrgic/Kossowsky/ Stieglitz/Vauth 
5 Gilmer TP, Dolder CR, Lacro JP, Folsom DP, Lindamer L, Garcia P, Jeste DV: Adherence to treatment with antipsychotic medication and health care costs among Medicaid beneficiaries with schizophrenia. Am J Psychiatry 2004;161:692-699.

-6 Nose M, Barbui C, Tansella M: How often do patients with psychosis fail to adhere to treatment programmes? A systematic review. Psychol Med 2003;33:1149-1160.

-7 Mintz AR, Dobson KS, Romney DM: Insight in schizophrenia: a meta-analysis. Schizophr Res 2003;61:75-88.

8 Perkins DO, Gu H, Weiden PJ, McEvoy JP, Hamer RM, Lieberman JA: Predictors of treatment discontinuation and medication nonadherence in patients recovering from a first episode of schizophrenia, schizophreniform disorder, or schizoaffective disorder: a randomized, double-blind, flexible-dose, multicenter study. J Clin Psychiatry 2008;69: 106-113.

$\checkmark 9$ Lincoln TM, Lullmann E, Rief W: Correlates and long-term consequences of poor insight in patients with schizophrenia. A systematic review. Schizophr Bull 2007;33:1324-1342.

-10 Williams CC: Insight, stigma, and post-diagnosis identities in schizophrenia. Psychiatry 2008;71:246-256.

11 Petrie KJ, Broadbent E, Kydd R: Illness perceptions in mental health: issues and potential applications. J Ment Health 2008;17:559564.

12 Holliday J, Wall E, Treasure J, Weinman J: Perceptions of illness in individuals with anorexia nervosa: a comparison with lay men and women. Int J Eat Disord 2005;37:50-56.

13 Lobban F, Barrowclough C, Jones S: The impact of beliefs about mental health problems and coping on outcome in schizophrenia. Psychol Med 2004;34:1165-1176.

14 Munson MR, Floersch JE, Townsend L: Attitudes toward mental health services and illness perceptions among adolescents with mood disorders. Child Adolesc Social Work J 2009;26:447-466.

15 Witteman C, Bolks L, Hutschemaekers G: Development of the Illness Perception Questionnaire Mental Health. J Ment Health 2011;20:115-125.

16 Lobban F, Barrowclough C, Jones S: Assessing cognitive representations of mental health problems. II. The Illness Perception Questionnaire for Schizophrenia: relatives' version. Br J Clin Psychol 2005;44:163-179.

-17 Fleming MP, Martin CR, Miles J, Atkinson J: The utility of the Illness Perception Questionnaire in the evaluation of mental health practitioners' perspectives on patients with schizophrenia. J Eval Clin Pract 2009;15: 826-831.

-18 Crisp AH, Gelder MG, Rix S, Meltzer HI, Rowlands OJ: Stigmatisation of people with mental illnesses. Br J Psychiatry 2000;177:4-7.

19 Kinderman P, Setzu E, Lobban F, Salmon P: Illness beliefs in schizophrenia. Soc Sci Med 2006;63:1900-1911.
20 Lobban F, Barrowclough C, Jones S: Assessing cognitive representations of mental health problems. I. The Illness Perception Questionnaire for Schizophrenia. Br J Clin Psychol 2005;44:147-162.

21 Broadbent E, Petrie KJ, Main J, Weinman J: The Brief Illness Perception Questionnaire. J Psychosom Res 2006;60:631-637.

22 Moss-Morris R, Weinman J, Petrie KJ, Horne R, Cameron LD, Buick D: The Revised Illness Perception Questionnaire. Psychol Health 2002;17:1-16.

23 Leventhal H, Nerenz DR, Steele DF: Illness representations and coping with health threats; in Baum A, Singer J (eds): A Handbook of Psychology and Health. Hillsdale/ NJ, Erlbaum, 1984.

24 Lobban F, Barrowclough C, Jones S: A review of the role of illness models in severe mental illness. Clin Psychol Rev 2003;23:171-196.

25 Watson PW, Garety PA, Weinman J, Dunn G, Bebbington PE, Fowler D, Freeman D, Kuipers E: Emotional dysfunction in schizophrenia spectrum psychosis: the role of illness perceptions. Psychol Med 2006;36:761770 .

26 Glattacker M, Bengel J, Jaeckel WH: The German version of the Illness Perception Questionnaire-Revised (IPQ-R): psychometric evaluation in patients with chronic somatic illness. Z Gesundheitspsychol 2009; 17:158-169.

27 Cohen J: Statistical Power Analysis for the Behavioral Sciences. New York, Psychology Press, 1988.

28 Kupper Z, Tschacher W: Lack of concordance between subjective improvement and symptom change in psychotic episodes. Br J Clin Psychol 2008;47:75-93.

29 Addington D, Addington J, Maticka-Tyndale E: Rating depression in schizophrenia. A comparison of a self-report and an observer report scale. J Nerv Ment Dis 1993;181:561565.

30 First MB, Gibbon M, Spitzer RL, Williams JBW: User's guide for the Structured Clinical Interview for DSM-IV Axis I Disorders - Research Version. New York, Biometrics Research, 1996.

-31 Brislin RW: Back-translation for cross-cultural research. J Cross Cult Psychol 1971;1: 185-216.

-32 Kay SR, Fiszbein A, Opler LA: The Positive and Negative Syndrome Scale (PANSS) for schizophrenia. Schizophr Bull 1987;13:261276.

33 Muller MJ, Rossbach W, Davids E, Wetzel H, Benkert O: Evaluation of standardized training for the Positive and Negative Syndrome Scale (PANSS). Nervenarzt 2000;71:195204.

34 Naber D, Moritz S, Lambert M, Pajonk FG, Holzbach R, Mass R, Andresen B: Improvement of schizophrenic patients' subjective well-being under atypical antipsychotic drugs. Schizophr Res 2001;50:79-88.
35 Muller MJ, Marx-Dannigkeit P, Schlosser R, Wetzel H, Addington D, Benkert O: The Calgary Depression Rating Scale for Schizophrenia: development and interrater reliability of a German version (CDSS-G). J Psychiatr Res 1999;33:433-443.

>36 Kuhner C, Burger C, Keller F, Hautzinger M: Reliability and validity of the Revised Beck Depression Inventory (BDI-II). Results from German samples. Nervenarzt 2007;78:651656.

-37 Amador XF, Strauss DH, Yale SA, Flaum MM, Endicott J, Gorman JM: Assessment of insight in psychosis. Am J Psychiatry 1993; 150:873-879.

>38 Birchwood M, Smith J, Drury V, Healy J, Macmillan F, Slade M: A self-report Insight Scale for psychosis: reliability, validity and sensitivity to change. Acta Psychiatr Scand 1994;89:62-67.

39 Horne R, Weinman J, Hankins M: The beliefs about medicines questionnaire: the development and evaluation of a new method for assessing the cognitive representation of medication. Psychol Health 1999;14:1-24.

40 Jonsdottir H, Friis S, Horne R, Pettersen KI, Reikvam A, Andreassen OA: Beliefs about medications: measurement and relationship to adherence in patients with severe mental disorders. Acta Psychiatr Scand 2009;119: 78-84.

41 Schmitt N: Uses and abuses of coefficient alpha. Psychol Assess 1996;84:350-353.

42 Briggs SR, Cheek JM: The role of factor analysis in the development of personality scales. J Pers 1986;54:106-148.

43 Grouven U, Bender R, Ziegler A, Lange S: Der Kappa-Koeffizient. Dtsch Med Wochenschr 2007;162:65-68.

44 Sedlmeier P: Jenseits des SignifikanztestRituals: Ergänzungen und Alternativen. MPR Online 1996;1:41-43.

45 Zubin J, Spring B: Vulnerability - a new view of schizophrenia. J Abnorm Psychol 1977;86: 103-126.

46 Onken SJ, Craig CM, Ridgway P, Ralph RO, Cook JA: An analysis of the definitions and elements of recovery: a review of the literature. Psychiatr Rehabil J 2007;31:9-22.

47 Cavelti M, Kvrgic S, Beck EM, Kossowsky J, Vauth R: The role of subjective illness beliefs and attitude toward recovery within the relationship of insight and demoralization among people with schizophrenia spectrum disorders. J Clin Psychol 2012, in press.

48 Chen SL, Tsai JC, Lee WL: Psychometric validation of the Chinese version of the Illness Perception Questionnaire-Revised for patients with hypertension. J Adv Nurs 2008; 64:524-534.

49 Cabassa LJ, Lagomasino IT, Dwight-Johnson M, Hansen MC, Xie B: Measuring Latinos' perceptions of depression: a confirmatory factor analysis of the Illness Perception Questionnaire. Cultur Divers Ethnic Minor Psychol 2008;14:377-384. 